OCU-PHYS 252

AP-GR 36

\title{
Relationship Between Solitonic Solutions of Five-Dimensional Einstein Equations
}

\author{
Shinya Tomizawa ${ }^{1}$, Hideo Iguchi $^{2}$ and Takashi Mishima ${ }^{2}$ \\ 1 Department of Mathematics and Physics, \\ Graduate School of Science, Osaka City University, \\ 3-3-138 Sugimoto, Sumiyoshi, Osaka 558-8585, Japan \\ 2 Laboratory of Physics, College of Science and Technology, Nihon University, \\ Narashinodai, Funabashi, Chiba 274-8501, Japan
}

(Dated: May 17, 2018)

\begin{abstract}
We give the relation between the solutions generated by the inverse scattering method and the Bäcklund transformation applied to the vacuum five-dimensional Einstein equations. In particular, we show that the two-solitonic solutions generated from an arbitrary diagonal seed by the Bäcklund transformation are contained within those generated from the same seed by the inverse scattering method.

PACS numbers: 04.50.+h 04.70.Bw
\end{abstract}




\section{INTRODUCTION}

Recently, the studies on higher dimensional black holes have much attention, since it has been predicted that they would be produced in a future linear collider [1]. In particular, a stationary black hole solution of Einstein equation has an important role in that it is expected to describe the classical equilibrium state and Hawking emission from it is considered to give us the signal of black hole production in the linear collider. By a lot of studies on higher dimensional black holes, it has been clarified that they have much complicated structure than in four-dimensions [2].

Some of higher dimensional stationary black hole solutions were found. As higher dimensional generalization of the Kerr black hole solution, the Myers-Perry black hole solution, which has an event horizon with spherical topology, was found [3]. Emparan and Reall found a black ring solution as the five-dimensional vacuum Einstein equation, which has an event horizon diffeomorphic to $S^{1} \times S^{2}$ [4] and describes a black hole rotating in the $S^{1}$ direction.

The black ring solution which is rotating only in $S^{2}$ direction was also found by two of the

present authors [5] by using one of the solitonic solution-generating techniques [6], so-called Bäcklund transformation. This is essentially the technique to generate new solutions of the Ernst equation from a known solution. In addition, it was shown by same authors that the black ring with $S^{1}$ rotation can be generated by the same solution generating technique [7].

As another solitonic technique, the inverse scattering technique [8] developed by Belinski and Zakharov is well known. This technique is essentially based on the fact that the Einstein's second-order nonlinear partial differential equations can be replaced with a pair of first-order linear partial differential equations called Lax pair. This method produces vacuum solutions from a certain known vacuum solution called a seed and succeeded in generation of a lot of four-dimensional solutions. In fact, as four-dimensional solutions the Kerr black hole solution, the multi Kerr black hole solutions and the Tomimastu-Sato solutions can be generated from the Minkowski seed and a physically interesting various solutions were also generated [9, 16].

Recently, some of higher-dimensional black hole/ring solutions have been generated by using the inverse scattering method. As an infinite number of static solutions of the five-dimensional vacuum Einstein equations with axial symmetry, the five-dimensional Schwarzschild solution and the static black ring solution were reproduced [11], which gave 
the first example of the generation of a higher-dimensional asymptotically flat black hole solution by the inverse scattering method. The Myers-Perry solution with single and double angular momenta were regenerated from the Minkowski [11, 12] and some unphysical seed [17], respectively. The black ring solutions with $S^{2}$ rotation was also reproduced by using this method from the Minkowski seed [12]. The $S^{1}$-rotating black ring solution was also reproduced from the Levi-Civita solution via the inverse scattering method by one of authors [18].

Thus, the inverse scattering method is a powerful formalism for solving systems of nonlinear partial differential equations such as Einstein equation. When we try to find a further new higher dimensional black hole/ring solution, it is important to know the relationship between solutions generated by the Bäcklund transformation used in Ref. [5] and ones generated by the inverse scattering method used in Ref. [12, 18] in that we can avoid the overlap of obtained solutions. Though the relationship between the four-dimensional solutions generated by the inverse scattering method and (the Bäcklund transformation was given) some Bäcklund transformations were considered in Ref. [13, 14] (see also Ref. 15, 16] about the relationship between the other generation-techniques), the relationship between higher dimensinoal solutions generated by these techniques dicussed here may be non-trivial and useful. In usual cases, in these solitonic generation-techniques a diagonal metric are often used as a seed since such a seed simplify the analysis. These techniques have a merit in that more complex solutions can be generated from simple solutions. This is why in this article, we investigate the relation between the solutions generated from a diagonal seed by both solitonic generation-techniques applied to five dimensions, and show that the two-solitonic solutions generated by Bäcklund transformation in Ref. [5] from an arbitrary diagonal seed coincide with two-solitonic solutions generated from the same seed by the inverse scattering method under the special normalization.

This article is organized as follows: In SecII we review the Bäcklund transformation developed by two of authors in Ref. [5] and the inverse scattering method applied to five dimensions by one of authors [12]. In Sec III, we show that the two-solitonic solutions generated by the former technique from a diagonal seed coincide with the special solutions generated by the latter technique from the same seed under the special normalization. 


\section{PRELIMINARY}

In this article, we consider the spacetimes which satisfy the following conditions: (1) five dimensions, (2) asymptotically flat spacetimes, (3) the solutions of vacuum Einstein equations, (4) having three commuting Killing vectors including one time-translational Killing vector and two axial Killing vectors. (5) having a single nonzero angular momentum component.

\section{A. Bäcklund transformation}

Here, we review the generation-technique of the five dimensional solution established by Ref. [5]. Under the conditions $(1)-(5)$, we can start the analysis from the following form of metric

$$
\begin{aligned}
d s^{2}= & e^{-T}\left[-e^{S}(d t-\omega d \phi)^{2}+e^{T+2 U_{1}} \rho^{2}(d \phi)^{2}\right. \\
& \left.+e^{2\left(\gamma+U_{1}\right)+T}\left(d \rho^{2}+d z^{2}\right)\right]+e^{2 T}(d \psi)^{2}
\end{aligned}
$$

Using this metric form the Einstein equations are reduced to the following set of equations,

$$
\begin{aligned}
& \text { (i) } \nabla^{2} T=0 \text {, } \\
& \text { (ii) }\left\{\begin{array}{l}
\partial_{\rho} \gamma_{T}=\frac{3}{4} \rho\left[\left(\partial_{\rho} T\right)^{2}-\left(\partial_{z} T\right)^{2}\right] \\
\partial_{z} \gamma_{T}=\frac{3}{2} \rho\left[\partial_{\rho} T \partial_{z} T\right]
\end{array}\right. \\
& \text { (iii) } \nabla^{2} \mathcal{E}_{S}=\frac{2}{\mathcal{E}_{S}+\overline{\mathcal{E}}_{S}} \nabla \mathcal{E}_{S} \cdot \nabla \mathcal{E}_{S} \\
& \text { (iv) }\left\{\begin{aligned}
\partial_{\rho} \gamma_{S} & =\frac{\rho}{2\left(\mathcal{E}_{S}+\overline{\mathcal{E}}_{S}\right)}\left(\partial_{\rho} \mathcal{E}_{S} \partial_{\rho} \overline{\mathcal{E}}_{S}-\partial_{z} \mathcal{E}_{S} \partial_{z} \overline{\mathcal{E}}_{S}\right) \\
\partial_{z} \gamma_{S} & =\frac{\rho}{2\left(\mathcal{E}_{S}+\overline{\mathcal{E}}_{S}\right)}\left(\partial_{\rho} \mathcal{E}_{S} \partial_{z} \overline{\mathcal{E}}_{S}+\partial_{\rho} \mathcal{E}_{S} \partial_{z} \overline{\mathcal{E}}_{S}\right),
\end{aligned}\right. \\
& \text { (v) }\left(\partial_{\rho} \Phi, \partial_{z} \Phi\right)=\rho^{-1} e^{2 S}\left(-\partial_{z} \omega, \partial_{\rho} \omega\right) \text {, } \\
& \text { (vi) } \gamma=\gamma_{S}+\gamma_{T} \text {, } \\
& \text { (vii) } U_{1}=-\frac{S+T}{2} \text {, }
\end{aligned}
$$

where the function $\Phi(\rho, z)$ is defined through the equation $(\mathrm{v})$ and the function $\mathcal{E}_{\mathcal{S}}$ is defined by $\mathcal{E}_{S}:=e^{S}+i \Phi$. It should be noted that $e^{S}$ and $\Phi$ corresponds to a gravitational potential and a twist potential. The equation (iii) is exactly the same as the Ernst equation in four 
dimensions [19]. The most nontrivial task to obtain new metrics is to solve the equation (iii) because of its nonlinearity. Here the method similar to the Neugebauer's Bäcklund transformation [20] or the Hoenselaers-Kinnersley-Xanthopoulos transformation [21] is used.

Following the procedure given by Castejon-Amenedo and Manko [22], for a static seed solution $e^{S^{(0)}}$ a new Ernst potential can be written in the form

$$
\mathcal{E}_{S}=e^{S^{(0)}} \frac{x(1+a b)+i y(b-a)-(1-i a)(1-i b)}{x(1+a b)+i y(b-a)+(1-i a)(1-i b)},
$$

where $x$ and $y$ are the prolate-spheroidal coordinates: $\rho=\sigma \sqrt{x^{2}-1} \sqrt{1-y^{2}}, z=\sigma x y$ with the ranges $1 \leq x$ and $-1 \leq y \leq 1$, and the functions $a$ and $b$ satisfy the following simple first-order differential equations

$$
\begin{aligned}
(\ln a)_{, x} & =\frac{1}{x-y}\left[(x y-1) S_{, x}^{(0)}+\left(1-y^{2}\right) S_{, y}^{(0)}\right], \\
(\ln a)_{, y} & =\frac{1}{x-y}\left[-\left(x^{2}-1\right) S_{, x}^{(0)}+(x y-1) S_{, y}^{(0)}\right], \\
(\ln b)_{, x} & =-\frac{1}{x+y}\left[(x y+1) S_{, x}^{(0)}+\left(1-y^{2}\right) S_{, y}^{(0)}\right], \\
(\ln b)_{, y} & =-\frac{1}{x+y}\left[-\left(x^{2}-1\right) S_{, x}^{(0)}+(x y+1) S_{, y}^{(0)}\right] .
\end{aligned}
$$

The corresponding expressions for the metric functions can be obtained by using the formulas shown by [22]. For the seed,

$$
\begin{aligned}
d s^{2}= & e^{-T^{(0)}}\left[-e^{S^{(0)}} d t^{2}+e^{-S^{(0)}} \rho^{2}(d \phi)^{2}\right. \\
& \left.+e^{2 \gamma_{(0)}-S^{(0)}}\left(d \rho^{2}+d z^{2}\right)\right]+e^{2 T^{(0)}}(d \psi)^{2} .
\end{aligned}
$$

a new solution is given by

$$
\begin{aligned}
d s^{2}= & -e^{S^{(0)}-T^{(0)}} \frac{A}{B}\left[d t-\left(2 \sigma e^{-S^{(0)}} \frac{C}{A}+C_{1}\right) d \phi\right]^{2} \\
& +\frac{B}{A} e^{-S^{(0)}-T^{(0)}} \sigma^{2}\left(x^{2}-1\right)\left(1-y^{2}\right) d \phi^{2}+e^{2 T^{(0)}} d \psi^{2} \\
& +e^{2 \gamma-S^{(0)}-T^{(0)}} \frac{B}{A} \sigma^{2}\left(x^{2}-y^{2}\right)\left(\frac{d x^{2}}{x^{2}-1}+\frac{d y^{2}}{1-y^{2}}\right),
\end{aligned}
$$


where $A, B$ and $C$ are defined by

$$
\begin{aligned}
& A:=\left(x^{2}-1\right)(1+a b)^{2}-\left(1-y^{2}\right)(b-a)^{2}, \\
& B:=[(x+1)+(x-1) a b]^{2}+[(1+y) a+(1-y) b]^{2}, \\
& C:=\left(x^{2}-1\right)(1+a b)[(1-y) b-(1+y) a]+\left(1-y^{2}\right)(b-a)[(x+1)-(x-1) a b] .
\end{aligned}
$$

To assure that the spacetime does not have global rotation, the constant $C_{1}$ is give by

$$
C_{1}=\frac{2 \sigma^{1 / 2} \alpha}{1+\alpha \beta} .
$$

\section{B. Inverse scattering Techniques}

We will give a summary of the inverse scattering method developed by Belinski and Zakharov [8] which is applied to five dimensions.

As in the previous subsection, we consider the asymptotically flat, five-dimensional stationary and axisymmetric vacuum spacetime with three commuting Killing vector fields $V_{(i)}(i=1,2,3)$ following the argument in [12, 18]. The commutativity of Killing vectors $\left[V_{(i)}, V_{(j)}\right]=0$ enables us to find a coordinate system such that $V_{(i)}=\partial / \partial x^{i}(i=1,2,3)$ and the metric is independent of the coordinates $x^{i}$, where $\left(\partial / \partial x^{1}\right)$ is the Killing vector field associated with time translation and $\left(\partial / \partial x^{2}\right),\left(\partial / \partial x^{3}\right)$ denote the spacelike Killing vector fields with closed orbits. We put $x^{1}=t, x^{2}=\phi$, and $x^{3}=\psi$. From the theorem in Ref. [23], in such a spacetime, the metric can be written in the canonical form [23] as

$$
d s^{2}=f\left(d \rho^{2}+d z^{2}\right)+g_{i j} d x^{i} d x^{j}
$$

where $f=f(\rho, z)$ and $g_{i j}=g_{i j}(\rho, z)$ are a function and an induced metric on the threedimensional space, respectively. Both of them depend only on the coordinates $\rho$ and $z$. Here it is the most convenient to choose the $3 \times 3$ matrix $g=(g)_{i j}$ as to satisfy the condition

$$
\operatorname{det} g=-\rho^{2}
$$

This is compatible with the vacuum Einstein equations $g^{i j} R_{i j}=0$, which reduces to $\left(\partial_{\rho}^{2}+\right.$ $\left.\partial_{z}^{2}\right)(-\operatorname{det} g)^{1 / 2}=0$. It follows from $R_{i j}=0$ that the matrix $g$ satisfies the solitonic equation

$$
\left(\rho g_{, \rho} g^{-1}\right)_{, \rho}+\left(\rho g_{, z} g^{-1}\right)_{, z}=0
$$


¿From the other components of the Einstein equations $R_{\rho \rho}-R_{z z}=0$ and $R_{\rho z}=0$, we obtain the equations which determine the function $f(\rho, z)$ for a given solution of the solitonic equation (9)

$$
\begin{aligned}
& (\ln f)_{, \rho}=-\frac{1}{\rho}+\frac{1}{4 \rho} \operatorname{Tr}\left(U^{2}-V^{2}\right), \\
& (\ln f)_{, z}=\frac{1}{2 \rho} \operatorname{Tr}(U V),
\end{aligned}
$$

where the $3 \times 3$ matrices $U(\rho, z)$ and $V(\rho, z)$ are defined by

$$
U:=\rho g_{, \rho} g^{-1}, \quad V:=\rho g_{, z} g^{-1}
$$

The integrability condition with respect to $f$ is automatically satisfied for the solution $g$ of Eq. (91). Note also that $R_{\rho \rho}+R_{z z}=0$ is consistent with the solution (91), (10) and (11).

Although our immediate goal is to solve the differential equations (9), it cannot be generally solved due to its non-linearity. But in analogy with the soliton technique, we can find the Lax pair for the matrix equations (92). We consider Schrödinger type equations for the $3 \times 3$ matrix $\psi(\lambda, \rho, z)$ as in four dimensions;

$$
D_{1} \psi=\frac{\rho V-\lambda U}{\lambda^{2}+\rho^{2}} \psi, \quad D_{2} \psi=\frac{\rho U+\lambda V}{\lambda^{2}+\rho^{2}} \psi
$$

where $\lambda$ is a complex spectral parameter independent of $\rho$ and $z$. The differential operators $D_{1}$ and $D_{2}$ are defined as

$$
D_{1}:=\partial_{z}-\frac{2 \lambda^{2}}{\lambda^{2}+\rho^{2}} \partial_{\lambda}, \quad D_{2}:=\partial_{\rho}+\frac{2 \lambda \rho}{\lambda^{2}+\rho^{2}} \partial_{\lambda},
$$

which can be shown to commute $\left[D_{1}, D_{2}\right]=0$. Note that Eq. (14) is invariant under the transformation $\lambda \rightarrow-\rho^{2} / \lambda$. Then the compatibility condition $\left[D_{1}, D_{2}\right] \psi=0$ reduces to the Einstein equations (9) with

$$
g(\rho, z)=\psi(0, \rho, z)
$$

It deserves to note that the Einstein's second-order nonlinear partial differential equations (9) are reduced to a pair of first-order linear partial differential equations (13).

Let $g_{0}, U_{0}, V_{0}$ and $\psi_{0}$ be particular solutions of Eq. (91) and (13). We shall call the known solution $g_{0}$ the seed solution. We are going to seek a new solution of the form

$$
\psi=\chi \psi_{0}
$$


which leads the following equations that the dressing matrix $\chi(\lambda, \rho, z)$ must satisfy

$$
\begin{aligned}
& D_{1} \chi=\frac{\rho V-\lambda U}{\lambda^{2}+\rho^{2}} \chi-\chi \frac{\rho V_{0}-\lambda U_{0}}{\lambda^{2}+\rho^{2}}, \\
& D_{2} \chi=\frac{\rho U+\lambda V}{\lambda^{2}+\rho^{2}} \chi-\chi \frac{\rho U_{0}+\lambda V_{0}}{\lambda^{2}+\rho^{2}} .
\end{aligned}
$$

In order for the solutions $g(\rho, z)$ to be real and symmetric, we impose the following conditions on the dressing matrix $\chi$,

$$
\bar{\chi}(\bar{\lambda}, \rho, z)=\chi(\lambda, \rho, z), \quad \bar{\psi}(\bar{\lambda}, \rho, z)=\psi(\lambda, \rho, z)
$$

and

$$
g=\chi\left(-\rho^{2} / \lambda, \rho, z\right) g_{0}{ }^{T} \chi(\lambda, \rho, z)
$$

where $\bar{\chi}$ and ${ }^{T} \chi$ denote complex conjugation and the transposition of $\chi$. ¿From Eqs. (16) and (19), the dressing matrix $\chi$ asymptotes to a unit matrix $\chi \rightarrow I$ as $\lambda \rightarrow \infty$.

The general $n$-soliton solutions for the matrix $g$ are generated due to the presence of the simple poles of the dressing matrix on the complex $\lambda$-plane:

$$
\chi=I+\sum_{k=1}^{n} \frac{R_{k}}{\lambda-\mu_{k}},
$$

where the matrices $R_{k}$ and the position of the pole $\mu_{k}$ depend only on the variables $\rho$ and $z$. Here and hereafter, the subscript $k, l$ counts the number of solitons. It is the characteristic feature of solitons that the dressing matrix $\chi$ is represented as the meromorphic function on the complex $\lambda$-plane. Pole trajectories $\mu_{k}(\rho, z)$ are determined by the condition that the left-hand side of Eq. (17) have no poles of second-order at $\lambda=\mu_{k}$, which yields following two differential equations for $\mu_{k}(\rho, z)$ :

$$
\mu_{k, z}=-\frac{2 \mu_{k}^{2}}{\mu_{k}^{2}+\rho^{2}}, \quad \mu_{k, \rho}=\frac{2 \rho \mu_{k}}{\mu_{k}^{2}+\rho^{2}},
$$

which are expressed by the solutions of the following quadratic equations

$$
\mu_{k}^{2}+2\left(z-w_{k}\right) \mu_{k}-\rho^{2}=0
$$

where $w_{k}$ are arbitrary constants. Solving Eq. (22), one can easily see

$$
\mu_{k}=w_{k}-z \pm \sqrt{\left(z-w_{k}\right)^{2}+\rho^{2}},
$$


where $w_{k}$ are arbitrary constants. Since the matrices $R_{k}$ are degenerate at the poles $R_{k} \chi^{-1}\left(\mu_{k}\right)=0$, which follows from the condition $\chi \chi^{-1}=I$ at $\lambda=\mu_{k}$, it is possible to write down the matrix elements of $R_{k}$ in the form

$$
\left(R_{k}\right)_{i j}=n_{i}^{(k)} m_{j}^{(k)}
$$

The fact that Eq. (17) has no residues at the poles $\lambda=\mu_{k}$ leads to obtain the vectors $m_{i}^{(k)}$ as

$$
m^{i(k)}=m_{0 j}^{(k)}\left[\psi_{0}^{-1}\left(\mu_{k}, \rho, z\right)\right]^{j i}
$$

where $m_{0 i}^{(k)}$ are arbitrary constants. The vectors $n_{i}^{(k)}$, on the other hand, are determined by the condition that Eq. (19) is regular at $\lambda=\mu_{k}$ as

$$
n_{i}^{(k)}=\sum_{l=1}^{n} \mu_{k}^{-1}\left(\Gamma^{-1}\right)_{k l} L_{i}^{(l)},
$$

where the vectors $L_{i}^{(k)}$ and the symmetric matrix $\Gamma_{k l}$ are given by

$$
\begin{aligned}
L_{i}^{(k)} & =m^{j(k)}\left(g_{0}\right)_{i j}, \\
\Gamma_{k l} & =\frac{m^{i(k)}\left(g_{0}\right)_{i j} m^{j(l)}}{\rho^{2}+\mu_{k} \mu_{l}},
\end{aligned}
$$

respectively. Therefore one can now find from Eq. (15), (16) and (20) that the matrix $g$ becomes

$$
\begin{aligned}
g_{i j}^{\text {(unphys })} & =\psi(0, \rho, z)_{i j} \\
& =\left(g_{0}\right)_{i j}-\sum_{k, l=1}^{n}\left(\Gamma^{-1}\right)_{k l} \mu_{k}^{-1} \mu_{l}^{-1} L_{i}^{(k)} L_{j}^{(l)} .
\end{aligned}
$$

This metric does not meet the condition $\operatorname{det} g=-\rho^{2}$, which we have denoted $g^{\text {(unphys) }}$. In order to satisfy the gauge condition $\operatorname{det} g=-\rho^{2}$, the metric should be appropriately normalized. One example is to normalize all the metric components by the same weight as

$$
g^{\text {(phys) }}=(-1)^{n / 3} \rho^{-2 n / 3}\left(\prod_{k=1}^{n} \mu_{k}^{2 / 3}\right) g^{\text {(unphys) }}
$$

where $g^{\text {(phys) }}$ is the metric which fulfills the condition $\operatorname{det} g=-\rho^{2}$. Actually, the fourdimensional Kerr solution is obtained similarly by the overall normalization as Eq. (30). 
Substituting the physical metric solution $g^{\text {(phys) }}$ given by Eq. (30) into Eq. (10) and (11), we obtain a physical value of $f$ as

$$
f=C_{0} f_{0} \rho^{-n(n-1) / 3} \operatorname{det}\left(\Gamma_{k l}\right) \prod_{k=1}^{n}\left[\mu_{k}^{2(n+2) / 3}\left(\mu_{k}^{2}+\rho^{2}\right)^{-1 / 3}\right] \cdot \prod_{k>l}^{n}\left(\mu_{k}-\mu_{l}\right)^{-4 / 3}
$$

where $C_{0}$ is an arbitrary constant, and $f_{0}$ is a value of $f$ corresponding to the seed $g_{0}$. In general, these solutions have two angular momentum component. In this article, we study the relationship between the solutions generated by the inverse scattering method applied to five-dimensions and those generated by the Bäcklund transformation in Ref. [5] which generates the only solutions with a single angular momentum component. So, we should compare the solutions which have a single angular momentum component generated by them with each other. As discussed in Ref. [12], since the the two-solitonic solution (30) with $n=2$ would not be regular on a certain part of an axis (as far as we choose a seed regular on it), it is suitable to normalize the metric so that $\left(g_{0}\right)_{33}$ is unchanged:

$$
g^{\text {(phys) }}=\left(\begin{array}{c|c}
\left(\prod_{k=1}^{n} \frac{\mu_{k}}{\rho}\right) g_{A B}^{\text {(unphys) }} & 0 \\
\hline 0 & \left(g_{0}\right)_{33}
\end{array}\right),
$$

where $A, B=1,2$. Here, we consider the two-soliton solution. We choose the sign of plus in Eq. (23) and take the constants $w_{1}=-w_{2}=-\sigma$.

\section{RELATION BETWEEN TWO-SOLITONIC SOLUTIONS}

In this section, we show that for a general diagonal seed solution which takes the form of

$$
d s^{2}=g_{1}^{\prime} d t^{2}+g_{2}^{\prime} d \phi^{2}+g_{3}^{\prime} d \psi^{2}+f^{\prime}\left(d \rho^{2}+d z^{2}\right)
$$

where $g_{1}^{\prime}, g_{2}^{\prime}$ and $g_{3}^{\prime}$ are functions of $\rho$ and $z$, and satisfy the constraint $g_{1}^{\prime} g_{2}^{\prime} g_{3}^{\prime}=-\rho^{2}$, the two-solitonic solutions generated by the inverse scattering method under the special normalization (32) coincide with ones generated by the Bäcklund transformation explained in Sec $\llbracket$ To do so, as a diagonal seed, instead of (33) it is sufficient to consider the following metric form,

$$
d s^{2}=-d t^{2}+g_{2} d \phi^{2}+g_{3} d \psi^{2}+f\left(d \rho^{2}+d z^{2}\right),
$$


where $g_{2}$ and $g_{3}$ are functions of $\rho$ and $z$, and satisfy the constraint $g_{2} g_{3}=\rho^{2}$ (In fact, starting with this form of the seed metric symplfies the proof).

The reason for this is explained as follows. Let us consider the conformal transformation of the two dimensional metric $g_{A B}(A, B=t, \phi)$ and the rescale of the $\psi \psi$-component with the determinant detg invariant ;

$$
g_{0}=\operatorname{diag}\left(-1, g_{2}, g_{3}\right) \rightarrow g_{0}^{\prime}=\operatorname{diag}\left(-\Omega, \Omega g_{2}, \Omega^{-2} g_{3}\right)
$$

where $\ln \Omega$ must be a harmonic function on the three-dimensional Euclid space in order to assure that the transformed metric is the solution of Eq.(9). (Since the metric function $f$ or $f^{\prime}$ is determined by only the three dimensional metric $g_{0}$ or $g_{0}^{\prime}$, we need not consider this for the present purpose) Under this transformation, the physical metric (32) is transformed as

$$
g=\left(\begin{array}{c|c}
g_{A B} & 0 \\
\hline 0 & g_{3}
\end{array}\right) \rightarrow g^{\prime}=\left(\begin{array}{c|c}
\Omega g_{A B} & 0 \\
\hline 0 & \Omega^{-2} g_{3}
\end{array}\right) .
$$

From this, we see that the transformation (35) of a seed commutes with the operation of putting two-solitons on the background. Therefore we can obtain the two-solitonic solution generated from a diagonal seed $g_{0}^{\prime}=\operatorname{diag}\left(g_{1}^{\prime}, g_{2}^{\prime}, g_{3}^{\prime}\right)$ such that the $t$-component is not -1 by the transformation (36) with $\Omega=g_{1}^{\prime}$ for the two-solitonic solution generated from the seed (34).

For the Bäcklund transformation, the same fact also holds, i.e. by the transformation (B5) of the seed, the solution generated is transformed as Eq.(36). Note that the seed functions for the metric (34) can be written in terms of $g_{2}$ as

$$
S^{(0)}=T^{(0)}=-\frac{1}{2} \ln \left(\frac{g_{2}}{\rho^{2}}\right) .
$$

The two-solitonic solution for the general seed metric with seed functions $S^{(0)}=$ $-1 / 2 \ln \left(g_{2} / \rho^{2}\right)$ and $T_{(0)}^{\prime} \neq-1 / 2 \ln \left(g_{2} / \rho^{2}\right)$ can be obtained from the solitonic solution of the seed (34) with the subsequent transformation (35) with $\Omega=e^{S^{(0)}-T_{(0)}^{\prime}}$. As a result, we can conclude that it is sufficient to assume the form of the diagonal seed as Eq.(34), i.e., the seed such that $\left(g_{0}\right)_{t t}=-1$ as far as we consider a diagonal seed solution.

To begin with, we show that for an arbitrary diagonal seed (34), the solutions of Eqs.(2) are given by

$$
a=-\alpha\left(\kappa_{1}+1\right) \sigma^{3 / 2} \frac{g_{2}^{1 / 2}(x+1)(1-y)}{\rho \psi_{2}\left[\rho, z, \mu_{2}\right]}, \quad b=-\beta \frac{\rho \psi_{2}\left[\rho, z, \mu_{1}\right]}{\left(\kappa_{2}-1\right) \sigma^{3 / 2}(x-1)(1-y) g_{2}^{1 / 2}},
$$


where $\psi_{2}[\rho, z, \lambda]$ is the $\phi \phi$-components of the solution $\psi$ of Eqs.(13) (we may assume the generating matrix $\psi_{0}[\rho, z, \lambda]$ to be diagonal $\psi_{0}[\rho, z, \lambda]=\operatorname{diag}\left(\psi_{1}[\rho, z, \lambda], \psi_{2}[\rho, z, \lambda], \psi_{3}[\rho, z, \lambda]\right)$ for a diagonal seed). $\alpha, \beta, \kappa_{1}$ and $\kappa_{2}$ are arbitrary constants.

From Eq.(37), the right hand side in the first equation of (2) is reduced to

$$
\frac{1}{x-y}\left[(x y-1) S_{, x}^{(0)}+\left(1-y^{2}\right) S_{, y}^{(0)}\right]=-\frac{1}{2(x-y)}\left[(x y-1)\left(\ln \frac{g_{2}}{\rho^{2}}\right)_{, x}+\left(1-y^{2}\right)\left(\ln \frac{g_{2}}{\rho^{2}}\right)_{, y}\right]
$$

On the other hand, using the first equation of (38), the left hand side in Eq. (2) becomes

$$
\begin{aligned}
(\ln a)_{, x}= & \frac{1}{2}\left(\ln g_{2}\right)_{, x}+\frac{1}{x+1}-(\ln \rho)_{, x}-\left(\ln \psi_{2}\left[\rho, z, \mu_{2}\right]\right)_{, x} \\
= & \frac{1}{2}\left(\ln g_{2}\right)_{, x}+\frac{1}{x+1}-(\ln \rho)_{, x}-\frac{\sigma^{2}}{\rho} x\left(1-y^{2}\right)\left(\ln \psi_{2}\left[\rho, z, \mu_{2}\right]\right)_{, \rho} \\
& -\sigma y\left(\ln \psi_{2}\left[\rho, z, \mu_{2}\right]\right)_{, z} .
\end{aligned}
$$

Let us note that the term containing $\left(\ln \psi\left[\rho, z, \mu_{2}\right]\right)_{, \rho}$ in the above equation is computed as

$$
\begin{aligned}
\left(\ln \psi_{2}\left[\rho, z, \mu_{2}\right]\right)_{, \rho} & =\left.\left(\ln \psi_{2}[\rho, z, \lambda]\right)_{, \rho}\right|_{\lambda=\mu_{2}}+\left.\left(\ln \psi_{2}[\rho, z, \lambda]\right)_{, \lambda}\right|_{\lambda=\mu_{2}} \cdot \mu_{2, \rho} \\
& =-\left.\frac{2 \mu_{2} \rho}{\rho^{2}+\mu_{2}^{2}}\left(\ln \psi_{2}[\rho, z, \lambda]\right)_{, \lambda}\right|_{\lambda=\mu_{2}}+\frac{\rho^{2}\left(\ln g_{2}\right)_{, \rho}+\rho \mu_{2}\left(\ln g_{2}\right)_{, z}}{\rho^{2}+\mu_{2}^{2}} \\
& +\left.\left(\ln \psi_{2}[\rho, z, \lambda]\right)_{, \lambda}\right|_{\lambda=\mu_{2}} \cdot \frac{2 \mu_{2} \rho}{\rho^{2}+\mu_{2}^{2}} \\
& =\frac{\rho^{2}\left(\ln g_{2}\right)_{, \rho}+\rho \mu_{2}\left(\ln g_{2}\right)_{, z}}{\rho^{2}+\mu_{2}^{2}}
\end{aligned}
$$

where we used Eq.(13) and (21). Similarly, the term containing $\left(\ln \psi\left[\rho, z, \mu_{2}\right]\right)_{, z}$ in Eq.(40) can be computed as

$$
\left(\ln \psi_{2}\left[\rho, z, \mu_{2}\right]\right)_{, z}=\frac{\rho^{2}\left(\ln g_{2}\right)_{, z}-\rho \mu_{2}\left(\ln g_{2}\right)_{, \rho}}{\rho^{2}+\mu_{2}^{2}}
$$

Therefore, using Eqs.(41) and (42), Eq.(40) becomes

$$
(\ln a)_{, x}=-\frac{1}{2(x-y)}\left[(x y-1)\left(\ln \frac{g_{2}}{\rho^{2}}\right)_{, x}+\left(1-y^{2}\right)\left(\ln \frac{g_{2}}{\rho^{2}}\right)_{, y}\right] .
$$

This coincides with the right hand side of Eq.(39), which implies that $a$ in Eq.(38) is a solution of the first equation in Eq.(2). Similarly, we can show a satisfies the second equation of (2) and that $b$ also satisfies the third and fourth equations of (2). As a result we see that the solutions (21) are given by Eqs.(38) for a diagonal seed whose $t t$-component is -1 .

Substituting Eq.(38) into Eqs.(44) and (15), we obtain the following general solution generated from a static seed solution,

$$
g_{t t}=-\frac{\tilde{A}}{\tilde{B}}, \quad g_{t \phi}=2 \sigma^{\frac{1}{2}} g_{2} \frac{\tilde{C}}{\tilde{B}}+C_{1} \frac{\tilde{A}}{\tilde{B}}, \quad g_{\phi \phi}=\frac{g_{t \phi}^{2}-\rho^{2}}{g_{t t}} .
$$


Here we have introduced new functions $\tilde{A}, \tilde{B}$ and $\tilde{C}$ defined as

$$
\begin{aligned}
& \tilde{A}=-\beta^{2} \psi_{2}\left[\mu_{1}\right]^{2} \psi_{2}\left[\mu_{2}\right]^{2}(1+y)^{2}+\sigma \alpha^{2} \beta^{2}\left(\kappa_{1}+1\right)^{2} g_{2} \psi_{2}\left[\mu_{1}\right]^{2}(x+1)^{2} \\
&+\sigma\left(\kappa_{2}-1\right)^{2} g_{2} \psi_{2}\left[\mu_{2}\right]^{2}(x-1)^{2}-\sigma^{2} \alpha^{2}\left(\kappa_{1}+1\right)^{2}\left(\kappa_{2}-1\right)^{2} g_{2}^{2}(1-y)^{2} \\
&+2 \sigma \alpha \beta\left(\kappa_{1}+1\right)\left(\kappa_{2}-1\right) g_{2} \psi_{2}\left[\mu_{1}\right] \psi_{2}\left[\mu_{2}\right]\left(x^{2}-y^{2}\right) \\
& \tilde{B}= \beta^{2} \psi_{2}\left[\mu_{1}\right]^{2} \psi_{2}\left[\mu_{2}\right]^{2}\left(1-y^{2}\right)+\sigma^{2} \alpha^{2}\left(\kappa_{1}+1\right)^{2}\left(\kappa_{2}-1\right)^{2} g_{2}^{2}\left(1-y^{2}\right) \\
&+\sigma \alpha^{2} \beta^{2}\left(\kappa_{1}+1\right)^{2} \psi_{2}\left[\mu_{1}\right]^{2} g_{2}\left(x^{2}-1\right)+\sigma\left(\kappa_{2}-1\right)^{2} \psi_{2}\left[\mu_{2}\right]^{2} g_{2}\left(x^{2}-1\right) \\
&+2 \sigma \alpha \beta\left(\kappa_{1}+1\right)\left(\kappa_{2}-1\right) g_{2} \psi_{2}\left[\mu_{1}\right] \psi_{2}\left[\mu_{2}\right]\left(x^{2}-y^{2}\right) \\
& \tilde{C}=-\beta\left(\kappa_{2}-1\right) \psi_{2}\left[\mu_{1}\right] \psi_{2}\left[\mu_{2}\right]^{2}(x+y)-\alpha \beta^{2}\left(\kappa_{1}+1\right) \psi_{2}\left[\mu_{1}\right]^{2} \psi_{2}\left[\mu_{2}\right](x-y) \\
&+\sigma \alpha^{2} \beta\left(\kappa_{2}-1\right)\left(\kappa_{1}+1\right)^{2} g_{2} \psi_{2}\left[\mu_{1}\right](x+y)+\sigma \alpha\left(\kappa_{1}+1\right)\left(\kappa_{2}-1\right)^{2} g_{2} \psi_{2}\left[\mu_{2}\right](x-y) .(4)
\end{aligned}
$$

Next, let us consider the solutions generated from the inverse scattering method. Under the special normalization (32), the two-soliton solution can be written in the following form:

$$
\begin{aligned}
& g_{t t}^{\text {(phys) }}=-\frac{G_{t t}}{\mu_{1} \mu_{2} \Sigma}, \quad g_{t \phi}^{\text {(phys) }}=-g_{2} \frac{\left(\rho^{2}+\mu_{1} \mu_{2}\right) G_{t \phi}}{\mu_{1} \mu_{2} \Sigma}, \quad g_{\phi \phi}^{(\text {phys })}=-g_{2} \frac{G_{\phi \phi}}{\mu_{1} \mu_{2} \Sigma}, \\
& g_{\psi \psi}^{\text {(phys) }}=g_{3}, \quad g_{\phi \psi}^{\text {(phys) }}=g_{t \psi}^{\text {(phys) }}=0,
\end{aligned}
$$

where the functions $G_{t t}, G_{t \phi}, G_{\phi \phi}$ and $\Sigma$ are defined as

$$
\begin{aligned}
G_{t t}= & -m_{01}^{(1) 2} m_{01}^{(2) 2} \psi_{2}\left[\mu_{1}\right]^{2} \psi_{2}\left[\mu_{2}\right]^{2}\left(\mu_{1}-\mu_{2}\right)^{2} \rho^{4}+m_{01}^{(1) 2} m_{02}^{(2) 2} g_{2} \mu_{2}^{2}\left(\rho^{2}+\mu_{1} \mu_{2}\right)^{2} \psi_{2}\left[\mu_{1}\right]^{2} \\
& +m_{01}^{(2) 2} m_{02}^{(1) 2} g_{2} \mu_{1}^{2}\left(\rho^{2}+\mu_{1} \mu_{2}\right)^{2} \psi_{2}\left[\mu_{2}\right]^{2}-m_{02}^{(1) 2} m_{02}^{(2) 2} g_{2}^{2} \mu_{1}^{2} \mu_{2}^{2}\left(\mu_{1}-\mu_{2}\right)^{2} \\
& -2 m_{01}^{(1)} m_{01}^{(2)} m_{02}^{(1)} m_{02}^{(2)} g_{2} \psi_{2}\left[\mu_{1}\right] \psi_{2}\left[\mu_{2}\right]\left(\rho^{2}+\mu_{1}^{2}\right)\left(\rho^{2}+\mu_{2}^{2}\right) \mu_{1} \mu_{2} \\
G_{\phi \phi}= & m_{01}^{(1) 2} m_{01}^{(2) 2} \mu_{1}^{2} \mu_{2}^{2}\left(\mu_{1}-\mu_{2}\right)^{2} \psi_{2}\left[\mu_{1}\right]^{2} \psi_{2}\left[\mu_{2}\right]^{2}+m_{02}^{(1) 2} m_{02}^{(2) 2} g_{2}^{2}\left(\mu_{1}-\mu_{2}\right)^{2} \rho^{4} \\
& -m_{01}^{(1) 2} m_{02}^{(2) 2} g_{2} \mu_{1}^{2} \psi_{2}\left[\mu_{1}\right]^{2}\left(\rho^{2}+\mu_{1} \mu_{2}\right)^{2}-m_{01}^{(2) 2} m_{02}^{(1) 2} g_{2} \mu_{2}^{2}\left(g_{2}-\mu_{2}\right)^{2}\left(\rho^{2}+\mu_{1} \mu_{2}\right)^{2}(51 \\
& +2 m_{01}^{(1)} m_{01}^{(2)} m_{02}^{(1)} m_{02}^{(2)} g_{2} \mu_{1} \mu_{2} \psi_{2}\left[\mu_{2}\right] \psi_{2}\left[\mu_{1}\right]\left(\rho^{2}+\mu_{1}^{2}\right)\left(\rho^{2}+\mu_{2}^{2}\right) \\
G_{t \phi}= & m_{01}^{(1)} m_{01}^{(2) 2} m_{02}^{(1)} \mu_{2}\left(\mu_{1}-\mu_{2}\right) \psi_{2}\left[\mu_{2}\right]^{2} \psi_{2}\left[\mu_{1}\right]\left(\rho^{2}+\mu_{1}^{2}\right) \\
& +m_{01}^{(1)} m_{02}^{(1)} m_{02}^{(2) 2} g_{2} \mu_{2}\left(\mu_{2}-\mu_{1}\right) \psi_{2}\left[\mu_{1}\right]\left(\rho^{2}+\mu_{1}^{2}\right) \\
& +m_{01}^{(1) 2} m_{01}^{(2)} m_{02}^{(2)} \mu_{1}\left(\mu_{2}-\mu_{1}\right) \psi_{2}\left[\mu_{1}\right]^{2} \psi_{2}\left[\mu_{2}\right]\left(\rho^{2}+\mu_{2}^{2}\right) \\
& +m_{01}^{(2)} m_{02}^{(1) 2} m_{02}^{(2)} \mu_{1} g_{2} \psi_{2}\left[\mu_{2}\right]\left(\rho^{2}+\mu_{2}^{2}\right)\left(\mu_{1}-\mu_{2}\right)
\end{aligned}
$$




$$
\begin{aligned}
\Sigma= & m_{01}^{(1) 2} m_{01}^{(2) 2} \psi_{2}\left[\mu_{1}\right]^{2} \psi_{2}\left[\mu_{2}\right]^{2}\left(\mu_{1}-\mu_{2}\right)^{2} \rho^{2}+m_{02}^{(1) 2} m_{02}^{(2) 2} g_{2}^{2}\left(\mu_{1}-\mu_{2}\right)^{2} \rho^{2} \\
& +m_{01}^{(1) 2} m_{02}^{(2) 2} g_{2} \psi_{2}\left[\mu_{1}\right]^{2}\left(\rho^{2}+\mu_{1} \mu_{2}\right)^{2}+m_{02}^{(1) 2} m_{01}^{(2) 2} g_{2} \psi_{2}\left[\mu_{2}\right]^{2}\left(\rho^{2}+\mu_{1} \mu_{2}\right)^{2} \\
& -2 m_{01}^{(1)} m_{01}^{(2)} m_{02}^{(1)} m_{02}^{(2)} g_{2} \psi_{2}\left[\mu_{1}\right] \psi_{2}\left[\mu_{2}\right]\left(\rho^{2}+\mu_{1}^{2}\right)\left(\rho^{2}+\mu_{2}^{2}\right),
\end{aligned}
$$

where the two functions $g_{2}$ and $g_{3}$ are given by Eq. (34).

In order for the metric to approach the Minkowski spacetime asymptotically, let us consider the coordinate transformation of the physical metric such that

$$
t \rightarrow t^{\prime}=t-C_{1} \phi, \quad \phi \rightarrow \phi^{\prime}=\phi
$$

where $C_{1}$ is a constant chosen to ensure the asymptotic flatness. We should note that the transformed metric also satisfies the supplementary condition $\operatorname{det} g=-\rho^{2}$. Under this transformation, the physical metric components become

$$
\begin{aligned}
& g_{t t}^{(\text {phys })} \rightarrow g_{t^{\prime} t^{\prime}}^{(\text {phys })}=g_{t t}^{(\text {phys })} \\
& g_{t \phi}^{(\text {phys })} \rightarrow g_{t^{\prime} \phi^{\prime}}^{(\text {phys })}=g_{t \phi}^{(\text {phys })}+C_{1} g_{t t}^{(\text {phys })}, \\
& g_{\phi \phi}^{(\text {phys })} \rightarrow g_{\phi^{\prime} \phi^{\prime}}^{\text {(phys) }}=g_{\phi \phi}^{(\text {phys })}+2 C_{1} g_{t \phi}^{(\text {phys })}+C_{1}^{2} g_{t t}^{(\text {phys })} .
\end{aligned}
$$

If we choose the parameters such that

$$
\begin{aligned}
m_{01}^{(1)} m_{01}^{(2)} & =\beta \\
m_{01}^{(2)} m_{02}^{(1)} & =\sigma^{\frac{1}{2}}\left(\kappa_{2}-1\right) \\
m_{01}^{(1)} m_{02}^{(2)} & =-\sigma^{\frac{1}{2}} \alpha \beta\left(\kappa_{1}+1\right), \\
m_{02}^{(1)} m_{02}^{(2)} & =-\sigma \alpha\left(\kappa_{1}+1\right)\left(\kappa_{2}-1\right), \\
C_{1} & =\frac{2 \sigma^{1 / 2} \alpha}{1+\alpha \beta}
\end{aligned}
$$

and use the prolate spherical coordinate $(x, y)$, we can confirm that the transformed metric coincides with the metric (44) generated by the technique used in Ref. [5] from a diagonal seed. In order to show the coincidence of the metrics, it is sufficient to check only two components $g_{t t}$ and $g_{t \phi}$ due to the supplementary condition $\operatorname{det} g=-\rho^{2}$ and the fact that the metric components $g_{\rho \rho}$ and $g_{z z}$ (or, $g_{x x}$ and $g_{y y}$ ) are determined by the three-dimensional metric $g_{i j}$. 


\section{SUMMARY AND DISCUSSION}

In this article, we studied the relation between the inverse scattering method and the Bäcklund transformation applied to five dimensions. We showed that the two-solitonic solution generated from an arbitrary diagonal seed by the Bäcklund transformation coincides with one generated from the same diagonal seed by the inverse scattering method under the special normalization (32). This implies that the five-dimensional solutions generated by the inverse scattering method contain the ones generated by the Bäcklund transformation used in Ref. 5] as concerned with the two-solitonic solutions generated from a diagonal seed. As clarified in the previous works [5, 7, 12, 18], if we choose the five-dimensional Minkowski or the Euclidean $C$-metric as a diagonal seed, we can obtain the black ring solution with a rotating two-sphere [5] or the black ring solution found by Emparan and Reall [4] as the two-solitonic solution, respectively. Therefore, we see that the previous works [12, 18] correspond to the special cases of the present result.

However, while the Bäcklund transformation used in Ref. [5] can generate solutions with a single angular momentum component at the most, the inverse scattering method can generate five-dimensional solutions with two angular momentum components, as discussed in Ref. [12, 18]. In fact, the Myers-Perry black hole solution with two angular momentum components was generated by the inverse scattering method [17]. It is expected that a new black ring solution with two angular momentum components may be generated by this method.

Though in this article, we focus on the two-solitonic solution generated from a diagonal seed by these techniques, we also expect solutions generated from non-diagonal seeds by both generation techniques or multi-solitonic solution (more than two) generated by them to be same if we choose the special normalization (32) in the inverse scattering method. It would be also interesting to deal with the generation of solutions in the five-dimensional Einstein-Maxwell equations with the same symmetries [24].

\section{Acknowledgments}

We thank Ken-ichi Nakao and Hideki Ishihara for continuous encouragement. HI is supported in part by Grant-in-Aid for Young Scientists (B) (No. 17740152) from Japanese 
Ministry of Education, Science, Sports, and Culture.

[1] T. Banks and W. Fischler, arXiv:hep-th/9906038, S. B. Giddings and S. D. Thomas, Phys. Rev. D 65, 056010 (2002); S. Dimopoulos and G. Landsberg, Phys. Rev. Lett. 87, 161602 (2001).

[2] M. I. Cai and G. J. Galloway, Class. Quant. Grav. 18, 2707 (2001); C. Helfgott, Y.Oz, and Y. Yanay, JHEP, 02, 025 (2006); G. J. Galloway and R.Schoen, arXive:gr-qc/0509107

[3] R. C. Myers and M. J. Perry, Annals Phys. 172, 304 (1986).

[4] R. Emparan and H. S. Reall, Phys. Rev. Lett. 88, 101101 (2002); R. Emparan and H. S. Reall, arXive hep-th/0608012.

[5] T. Mishima and H. Iguchi, Phys. Rev. D73, 044030, (2006); H. Iguchi and T. Mishima, Phys. Rev. D 74, 024029 (2006).

[6] J. Castejon-Amenedo and V. S. Manko, Phys. Rev. D 41, 2018 (1990).

[7] H.Iguchi and T.Mishima, Phys. Rev. D 73, 121501(R) (2006).

[8] V. A. Belinskii and V. E. Zakharov, Sov. Phys. JETP 50, 1 (1979); V. A. Belinskii and V. E. Zakharov, Sov. Phys. JETP 48, 985 (1978).

[9] V. A. Belinski and E. Verdaguer, Gravitational Solitons (CambridgeUniversity Press, Cambridge, England, 2001).

[16] H. Stephani, D. Kramer, M. MacCallum, C. Hoenselaers and E. Herlt, Exact solutions of Einstein's Field Equations, 2nd ed. (Cambridge University Press, Cambridge, 2003).

[11] T. Koikawa, Prog. Theor. Phys. 114, 793 (2005).

[12] S. Tomizawa, Y. Morisawa, Y Yasui, Phys. Rev. D73, 064009 (2006).

[13] M. Gürses, Inverse Scattering, Differential Geometry, Einstein Maxwell Solitons and One Soliton Backlund Transformations in Solutions of Einstein's Equations: Techniques and Results Edited by C. Hoenselaers and W. Dietz, (Springer-Verlag, Berlin, 1984).

[14] M. Gürses, Phys.Rev.Lett. 51, 1810 (1983); M. Gürses, Phys.Rev.D 30, 486(1984).

[15] C. M. Cosgrove, J.Math.Phys. 21, 2417 (1980).

[16] H. Stephani, D. Kramer, M. MacCallum, C. Hoenselaers and E. Herlt, Exact solutions of Einstein's Field Equations, 2nd ed. (Cambridge University Press, Cambridge, 2003).

[17] A. A. Pomeransky, Phys. Rev. D 73, 044004 (2006). 
[18] S. Tomizawa and M. Nozawa, Phys. Rev. D 73, 124034 (2006).

[19] F. J. Ernst, Phys. Rev. 167, 1175 (1968).

[20] G. Neugebauer, J. Phys. A 13, L19 (1980).

[21] C. Hoenselaers, W. Kinnersley and B. C. Xanthopoulos, J. Math. Phys. 20, 2530 (1979).

[22] J. Castejon-Amenedo and V. S. Manko, Phys. Rev. D 41, 2018 (1990).

[23] R. Emparan, H. S. Reall, Phys. Rev. D 65, 084025 (2002).

[24] A. Eriş, M. Gürses and A. Karasu, J.Math.Phys. 25, 1489(1984). 